\title{
"Sleeve" Sinus of Valsalva Repair in Patients with Acute Type A Aortic Dissection
}

\author{
Laichun Song, MD, ${ }^{1}$ Yang Gao, MD, ${ }^{2}$ Ming Xu, MD,${ }^{1}$ Bo Wang, MD,${ }^{1}$ Xiaoyong Li, MD,${ }^{1}$ Xiao Wang, MD
}

${ }^{1}$ Department of Cardiac Surgery, Asia Heart Hospital, Wuhan, P.R. China, Affiliated Wuhan University of Science and Technology, Wuhan, P.R. China; ${ }^{2}$ Department of Anesthesiology, Asia Heart Hospital, Wuhan, P.R. China

\section{ABSTRACT}

Purpose: The optimal surgical strategy of aortic root in acute type A aortic dissection (ATAAD) is controversial. The aim of this study was to evaluate the feasibility and safety of "Sleeve" Sinus of Valsalva repair for AAD limited to the noncoronary sinus or partial left and right coronary sinus without involvement coronary artery ostia.

Methods: From September 2016 to March 2019, 20 patients with $\mathrm{AAD}$ involving non-coronary sinus or partial left and right coronary sinus Valsalva underwent "Sleeve" Sinus of Valsalva repair. A tailored Dacron patch was inserted into the dissected layers and two Dacron strips were placed inside and outside of the aorta, and the new five-layers root was sutured with $3 / 0$ prolene continuous stitches. The artificial vessel was reversed about $1 \mathrm{~cm}$ and anastomosed with the reconstructed root. Then, the reversed artificial vessel was pulled and anastomosed with the Dacron strip of the new proximal aorta.

Results: There was no early death in hospital and one death occurred during the 30-day postoperative period. Re-thoracotomy due to bleeding was necessary in only one patient and no bleeding was related to the proximal anastomosis. The postoperative drainage was $390.5 \pm 229.3 \mathrm{~mL}$. During follow up, the echocardiography showed the normal sinus of Valsalva and aortic valvular function. Computed tomography angiography showed normal aortic root without endovascular leak or dissection around the sinus of Valsalva. All patients were free from reoperation.

Conclusions: "Sleeve" Sinus of Valsalva repair with Dacron patch for aortic dissection limited to the non-coronary sinus or partial left and right coronary sinus without involvement coronary artery ostia was technically feasible and safe.

\section{INTRODUCTION}

Acute type A aortic dissection (ATAAD) is a life-threatening disease with contemporary perioperative morality rates of $13 \%$ to $17 \%$ [Mussa 2016; Pape 2015]. The dissection flap in the ascending aorta often extends into the sinus segment, resulting in aortic regurgitation (AR) [Movsowitz 2000]. The aortic root anatomy, including aortic valve and sinus segment,

Received fanuary 23, 2021; accepted March 2, 2021.

Correspondence: Xiao Wang, MD, Department of Cardiac Surgery, Asia Heart Hospital, Wuban, P.R. China, No.753 Finghan Road, Hankou District, Wuban, P.R. China, 430022 (e-mail: doctorslc@hotmail.com). is normal in most patients before experiencing ATAAD. In addition, the aortic apparatus, including the aortic annulus and leaflets, are not involved in most cases, which makes the aortic root reconstruction possible to preserve the aortic leaflets and function. Thus, various surgical techniques, including the external, internal, or intramural reinforcement with prosthetic, biologic, or autologous materials, have been applied to reconstruct the aortic root to spare the aortic leaflets [Rylski 2014; Tang 2017; Fleischman 2018]. However, there remain significant complications during the long-term follow up, including progressive dilatation of the preserved aortic root, formation of proximal false aneurysm, and progression of aortic regurgitation (AR) [Malvindi 2013; Ro 2013; Zierer 2007; Estrera 2007]. To prevent these events, total aortic root reconstruction or replacement including David, Yacoub and Bentall procedure may be performed, which could be burdened by the complex surgical manipulation and injury risk of coronary artery [David 1995; Komiya 2008].

The technique of "Sleeve" Sinus of Valsalva repair has been applied in our center for several years with excellent clinical results. The purpose of this study was to introduce the novel technique of "Sleeve" Sinus of Valsalva repair to preserve the aortic valve and to evaluate the feasibility and safety of the approach for proximal re-approximation.

\section{MATERIALS AND METHODS}

This study was approved by the Institutional Review Board of Wuhan Asia Heart Hospital and was in compliance with the Health Insurance Portability and Accountability Act regulations and the Declaration of Helsinki. The institutional review board waived the need for individual patient consent.

Patient selection: From September 2016 through March 2019, 20 consecutive patients with acute type A aortic dissection (ATAAD) underwent "Sleeve" Sinus of Valsalva repair combined with total arch replacement and stented elephant trunk implantation or hemiarch replacement in our center. Multi slice spiral computed tomography angiography (MSCT) and three dimensional reconstruction were routinely performed in all patients to assess the maximal diameters of each segment of the aorta, including the aortic root. Transthoracic echocardiography (TTE) and transesophageal echocardiography was performed in all patients to evaluate aortic regurgitation and other valvular dysfunction, to identify the location of intimal tears and the presence of coronary involvement. The location of intimal tears and extent of dissection in the aortic root was confirmed with intraoperative 


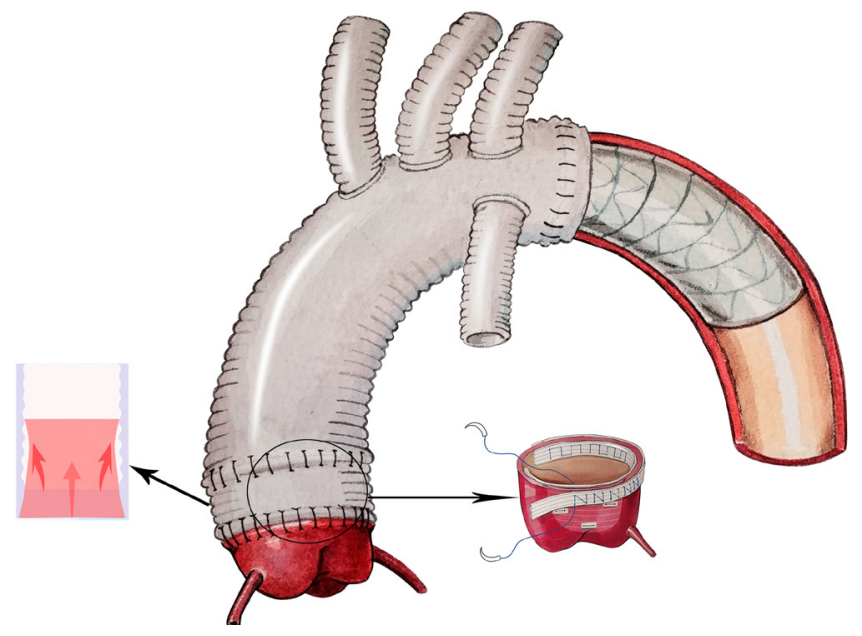

Figure 1. The rightward pointing arrow: A tailored Dacron patch was inserted into the dissected layers and a mattress stitch was used to fix the patch. Two $4 \mathrm{~mm}$ Dacron strips were placed inside and outside of the aorta and the new five-layers root was sutured with $3 / 0$ prolene continuous stitches. The leftward pointing arrow: "Sleeve" technique not only reinforced the anastomosed, but also reduced the tension of anastomosis when the aorta contracting and relaxing, which may reduce the bleeding and residual dissection in the sinus of Valsalva.

findings. The technique of "Sleeve" Sinus of Valsalva repair is performed in the patients with the diameter of aortic sinus $<45 \mathrm{~mm}$, AI without diseased leaflets, dissection limited in the non-coronary sinus or partial left and right coronary sinuses without involvement coronary artery ostia.

Surgical techniques: All patients underwent surgical intervention within 48 hours of clinical onset by the same surgeon. Procedures were performed under general anesthesia and via a standard median sternotomy. CPB was established by cannulating in the femoral artery and a dual-stage atriocaval cannula was used in the right atrium. Cerebral protection was established through the innominate artery for antegrade cerebral perfusion or the superior vena cava for retrograde cerebral perfusion. The innominate artery, left common carotid artery, and the LSA were dissected and exposed. Myocardial protection instituted with antegrade cold Del-Nido cardioplegia delivered antegrade. During the procedure, if there was no intimal tear in the arch, only the ascending aorta and/or hemiarch replacement was performed; if there was a tear in the arch and descending aorta, the ascending aorta and total arch replacement combined with stented elephant trunk implantation under direct visualization was performed. During cooling, aortic root procedures were performed, according to the extent of aortic dissection and pathologic status of the aortic valve.

The ascending aorta was transected circumferentially at approximately $5 \mathrm{~mm}$ above the STJ. All the thrombus in the dissected aortic layers were wiped out without destroying both the intima and adventitia. A tailored Dacron patch was inserted into the dissected layers and a mattress stitch was used to fix the patch at the bottle of the sinus. Two $4 \mathrm{~mm}$ Dacron strips were placed inside and outside of the aorta, and the new five-layers root was sutured with $3 / 0$ prolene continuous

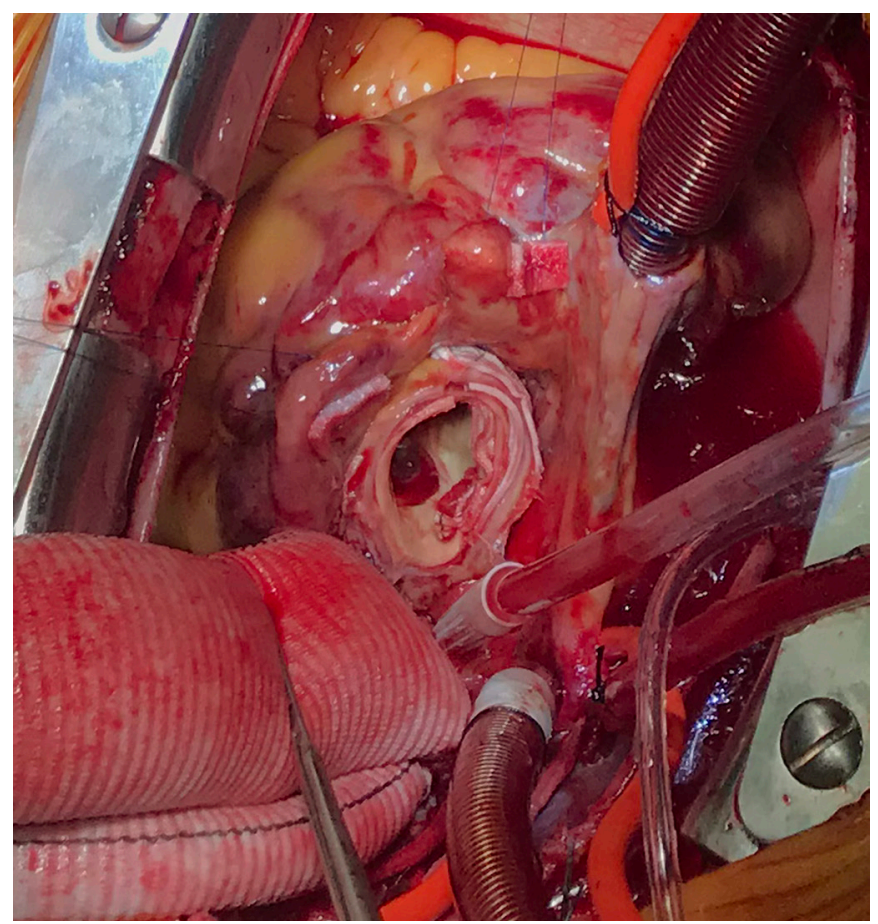

Figure 2. The " $Z$ " shape suture line was shown when reconstructing the sinus of Valsalva. "Sleeve" technique was used to reconstruct the aortic root. This technique reduced the tension of anastomosis when the aorta contracting and relaxing, which may reduce the bleeding and residua dissection in the sinus of Valsalva.

stitches ("Z" shape suture line) (Figures 1 and 2). To prevent interference of the coronary flow, the inner felt was frequently trimmed and secured at each orifice level of the coronary artery. Then the reconstructed root was anastomosed with artificial vessel. The artificial vessel was reversed about $1 \mathrm{~cm}$ and anastomosed with the reconstructed root. Then, the reversed artificial vessel was pulled and anastomosed with the Dacron strip of the new proximal aorta ("Sleeve" technique, Figure 2). However, this technique is not recommended if any of the following conditions are present: Marfan syndrome, the dissection involving two or more sinuses of Valsalva, a root aneurysm $(>4.5 \mathrm{~cm})$, and known connective tissue disorders.

Follow up: The durability of the root repair was assessed by follow-up transthoracic echocardiography and computed tomographic angiography 6 and 12 months postoperatively and annually thereafter.

Data management and statistical analysis: The data were managed and analyzed by using SPSS19.0. Data are expressed as mean \pm standard deviation for continuous variables and as median and range for categoric variables.

\section{RESULTS}

\section{Demographic and clinical characteristics}

The mean age was $61.65 \pm 9.54$ years (ranges from 49 to 80 years). Among them, 13 patients were male, and seven 


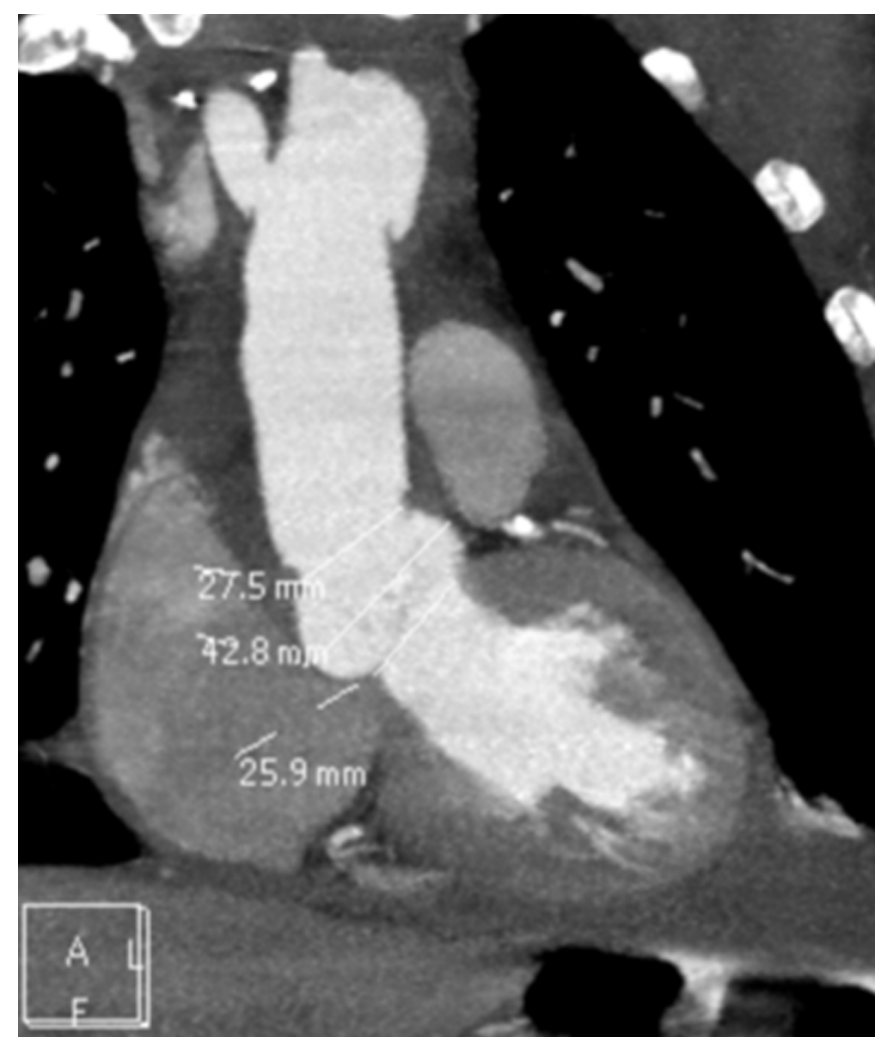

Figure 3. The computed tomography angiography showed normal aortic root without endovascular leak or dissection around the sinus of Valsalva.

patients were female. According to the echocardiography, the LVEF ranged from 40-60\% (mean $50.97 \pm 3.41$ ), and aortic regurgitation and mitral regurgitation were observed in 15 cases (75\%) and two cases (10\%), respectively. Four (20\%) patients had impaired renal function.

\section{Preoperative profiles}

All patients underwent "Sleeve" Sinus of Valsalva repair to preserve the aortic valve and no patient needed the Bentall procedure. Two patients (10\%) who had coronary artery malperfusion underwent CABG with saphenous vein graft. The most frequent involvement of the coronary artery was the right coronary artery. Two patients performed mitral annuloplasty with a strip of autogenous pericardium for the normal leaflets without anatomical dysfunction. The cardiopulmonary bypass time was $207.35 \pm 45.89$ minutes, cross-clamp time was $133.75 \pm 42.15$ minutes, and circulatory arrest time was $23.28 \pm 5.56$ minutes.

There was no patient death in hospital. Re-sternotomy, due to bleeding, was necessary in only one patient and no bleeding was related to the proximal anastomosis of the conduit. The mean size of sinuses of Valsalva was $3.52 \pm 1.26 \mathrm{~cm}$ after the procedure. Mild aortic regurgitation was observed in three patients, and there was no AR in other patients. The postoperative drainage was few $(390.5 \pm 229.3 \mathrm{~mL})$. The mean intubation time was $8.03 \pm 3.3$ hours, and ICU stay was
$2.69 \pm 0.13$ days. No stroke and persistent neurologic dysfunction occurred. Delirium was observed in one patient and recovered 5 th day after the procedure.

\section{Results from follow up}

Patient follow up was confirmed by a schedule of recent visits or by telephone, and follow up was complete in all patients. Patients were followed up every three months for the first year with echocardiography to detect the aortic root. Computed tomographic angiography was scheduled for each patient before discharge, every 6 months for the first year, and annually thereafter. The mean follow-up time was $21.3 \pm 8.3$ months (ranged from nine to 39 months). The echocardiography showed the normal sinus of Valsalva and aortic valvular function. The computed tomography angiography showed normal aortic root without endovascular leak or dissection around the sinus of Valsalva (Figure 3). All patients were free from reoperation.

\section{DISCUSSION}

Spontaneous acute type A aortic dissection is a rare and life-threatening condition. Surgical intervention is the optimal strategy for these patients, despite the association with high mortality and morbidity for the complex surgical manipulations. Complete or valve sparing aortic root replacement has been the most common procedure for this disease. However, there is no clear consensus about the surgical strategy for patients with the dissection limiting to the non or right coronary sinus. Because the other sinus of the Valsalva, aortic cusps, and sinotubular junction may be fairly intact in these patients, aortic root reconstruction may be possible to preserve the aortic valve.

In 1995, David [David 1995] first performed the partial aortic root repair to correct the dilated sinotubular junctions and dissected noncoronary sinus in abnormal or mild dilated aortic sinuses. Subsequently, various modifications to spare the aortic valve have been reported in patients with ATAAD [Rylski 2014; Tang 2017; Komiya 2008; Charitos 2009; Rylski 2013]. Komiya et al. [Komiya 2008] reported that the modification of partial aortic root remodeling was performed by fixing a U-shaped Dacron patch in the sinus. In their study, when the dissection involved right coronary sinus, the ostium of right coronary artery was reimplanted to the patch. Tang et al [Tang 2017] reported 151 patients with acute type A aortic dissection who underwent modified sandwich repair of aortic root with excellent long-term results. The modified sandwich technique using Teflon felt can be successfully performed in most patients with acute type A aortic dissection and is associated with low in-hospital mortality and low root reoperation rates in the long run. However, these techniques have some potential risk for dilatation of aortic root, aortic valve insufficiency, and pseudoaneurysm. Thus, to reduce the perioperative mortality rates and improve the long-term outcomes, an appropriate proximal aortic root procedure should be performed in selected patients with ATAAD. 
The principles for successful aortic root reconstruction are thorough elimination of dissection in the sinus of Valsalva, no aortic root bleeding during or after the procedure, and prevention of aortic regurgitation caused by aortic root dilatation and residual aortic sinus dissection or pseudoaneurysm during the long run [Geirsson 2007; Yang 2019]. Many surgical techniques with or without polytetrafluoroethylene felt bolsters or biological glue can be used to reconstruct the aortic to preserve aortic valve. However, the incidence of bleeding after the procedure were very high, which needs re-exploration, and reoperation should be performed for aortic pseudoaneurysm during the follow up [Yang 2019]. Fortunately, in our series, re-sternotomy due to bleeding was necessary in only one patient, and no bleeding was related to the proximal anastomosis because placing the Dacron strips external and internal to the sinus of Valsalva wall and " $Z$ " shape suture line were very effective at preventing tears and bleeding from the needle holes in the dissected tissue. Furthermore, "Sleeve" technique not only reinforced the anastomosed, but also reduced the tension of anastomosis when the aorta contracting and relaxing, which may reduce the bleeding and residual dissection in the sinus of Valsalva (Figure 1, the leftward pointing arrow). In addition, the cardiac surgeon could palpate the reconstructed sinus of Valsalva wall to estimate the sinus of Valsalva dissection eliminated or not. This procedure does not demand advanced techniques and extra manipulations, thereby reducing the operative complexity and shortening the operative time. Conversely, this procedure may raise concerns, regarding pseudoaneurysm and late aortic regurgitation owing to aortic root dilatation of the reconstructive sinus or abnormal aortic cusp motion. During our follow up, there was no pseudoaneurysm or aortic regurgitation, however, the effectiveness and safety of this procedure should be confirmed through long-term, follow-up studies.

\section{CONCLUSION}

In conclusion, "Sleeve" Sinus of Valsalva repair with Dacron patch for aortic dissection limited to the noncoronary sinus or partial left and right coronary sinus without involvement coronary artery ostia was technically feasible and safe because of the satisfactory early results regarding reconstructing the sinus of Valsalva and preserving aortic valve function. A long-term, follow up is mandatory for the screening of late complications.

\section{ACKNOWLEDGEMENT}

Funding statement: This work was supported by Health and Family Planning Commission of Wuhan municipality scientific research project (WJ2018H0041, WJ2018H0047).
REFERENCES

Charitos EI, Stierle U, Sievers HH, et al. 2009. Valve-sparing aortic root remodeling with partial preservation of the intact native aortic sinuses. Eur J Cardiothorac Surg. 36(3):589-591.

David TE, Feindel CM, Bos J. 1995. Repair of the aortic valve in patients with aortic insufficiency and aortic root aneurysm. J Thorac Cardiovasc Surg. 109(2):345-351.

Estrera AL, Miller CC III, Villa MA, et al. 2007. Proximal reoperation after repaired acute type A aortic dissection. Ann Thorac Surg. 83 (5):1603-9.

Fleischman F, Elsayed RS, Cohen RG, et al. 2018. Selective Aortic Arch and Root Replacement in Repair of Acute Type A Aortic Dissection. Ann Thorac Surg. 105(2):505-512.

Geirsson A, Bavaria JE, Swarr D, et al. 2007. Fate of the residual distal and proximal aorta after acute type a dissection repair using a contemporary surgical reconstruction algorithm. Ann Thorac Surg. 84 (6):1955-64.

Komiya T, Tamura N, Sakaguchi G, et al. 2008. Modified partial aortic root remodeling in acute type A aortic dissection. Interact Cardiovasc Thorac Surg. 8(3):306-309.

Malvindi PG, van Putte BP, Sonker U, et al. 2013. Reoperation after acute type A Aortic dissection repair: a series of 104 patients. Ann Thorac Surg. 95 (3):922-8.

Movsowitz HD, Levine RA, Hilgenberg AD, et al. 2000. Transesophageal echocardiographic description of the mechanisms of aortic regurgitation in acute type A aortic dissection: implications for aortic valve repair. J Am Coll Cardiol. 36(3):884-890.

Mussa FF, Horton JD, Moridzadeh R, et al. 2016. Acute aortic dissection and intramural hematoma: a systematic review. JAMA. 316 (7):754-63.

Pape LA, Awais M, Woznicki EM, et al. 2015. Presentation, Diagnosis, and Outcomes of Acute Aortic Dissection: 17-Year Trends from the International Registry of Acute Aortic Dissection. Journal of the American College of Cardiology. Jul 28. 66(4):350-358.

Ro SK, Kim JB, Hwang SK, et al. 2013. Aortic root conservative repair of acute type A aortic dissection involving the aortic root: fate of the aortic root and aortic valve function. J Thorac Cardiovasc Surg. 146 (5):1113-8.

Rylski B, Bavaria JE, Milewski RK, et al. 2014. Long-term results of neomedia sinus valsalva repair in 489 patients with type A aortic dissection. Ann Thorac Surg. 98(2):582-589.

Rylski B, Beyersdorf F, Blanke P, et al. 2013. Supracoronary ascending aortic replacement in patients with acute aortic dissection type A: what happens to the aortic root in the long run? J Thorac Cardiovasc Surg. $146(2): 285-90$.

Tang Y, Liao Z, Han L, Tang H, et al. 2017. Long-term results of modified sandwich repair of aortic root in 151 patients with acute type A aortic dissection. Interact CardioVasc Thorac Surg. 25 (1):109-13.

Yang B, Norton EL, Hobbs R, et al. 2019. Short- and long-term outcomes of aortic root repair and replacement in patients undergoing acute type A aortic dissection repair: Twenty-year experience. J Thorac Cardiovasc Surg. 157(6):2125-2136.

Zierer A, Voeller RK, Hill KE, et al. 2007. Aortic enlargement and late reoperation after repair of acute type A aortic dissection. Ann Thorac Surg. 84 (2):479-87. 